\title{
Significado de la transición infancia-adolescencia- juventud en personas con enfermedad renal crónica
}

\author{
Meaning of childhood adolescence youth transition \\ in people with chronic kidney disease
}

\section{Significado da transição infância, adolescência e juventude em pessoas com doença renal crônica}

Flor Neyfy Botina Muñoz ${ }^{1}$, Luis Alexander Lovera Montilla ${ }^{2}$,

Luz Angela Argote Oviedo ${ }^{3}$, Jaime Manuel Restrepo Restrepo ${ }^{4}$

${ }^{1}$ Enfermera Especialista en Enfermería Nefrológica, Mg. Enfermería con énfasis en Cuidado al Niño. Profesora Asociada, Programa de Enfermería Universidad Libre. Cali, Colombia.

${ }^{2}$ Enfermero Especialista en Enfermería Neonatal, Mg. Administración en Salud. Profesor Asociado, Escuela de Enfermería Universidad del Valle. Cali, Colombia. luis.alexander.lovera@correounivalle.edu.co

${ }^{3}$ Enfermera Especialista en Enfermería Materno Perinatal, Mg. Enfermería con énfasis Materno Infantil, Profesora Titular Escuela de Enfermería Universidad del Valle. Cali, Colombia. luargote@gmail.com

${ }^{4}$ Nefrólogo Pediatra. Jefe del Servicio de Nefropediatría Fundación Clínica Valle del Lili. Cali, Colombia. restrepojaime@hotmail.com

Cómo citar este artículo en edición digital: Botina Muñoz, F. N., Lovera Montilla, L. A., Argote Oviedo, L. A., \& Restrepo Restrepo, J. M. (2018). Significado de la transición infancia-adolescencia-juventud en personas con enfermedad renal crónica.

Cultura de los Cuidados (Edición digital), 22(50). Recuperado de http://dx.doi.org/10.14198/cuid.2018.50.04

Correspondencia: Flor Neyfy Botina Muñoz Calle 50, No. 80 - 110. Ciudad Real. Cali, Colombia.

Correo electrónico: florbotina@gmail.com

Recibido: 21/06/2017; Aceptado: 02/11/ 2017

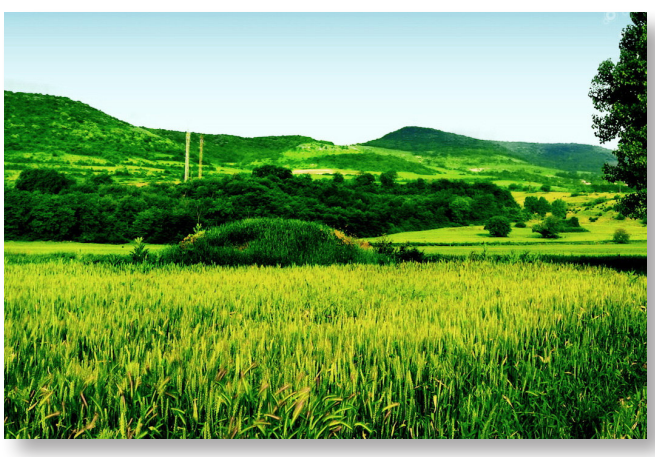

ABSTRACT

Introduction: The transition to youth in children and adolescents with chronic kidney disease (CKD) brings many changes that nursing professionals must incorporate in care protocols for health promotion and prevention of kidney disease.

Objective: To deepen, in people on dialysis therapy, the meaning of have lived the transition childhood, adolescence, youth with CKD.
Methodology: Qualitative study, following Grounded Theory in data. Nine participants aged between 18 and 24 years. The data collection was carried out through in-depth interviews and under the criteria of credibility, auditability and transferability.

Findings: Through codification data, theoretical sampling and the constant comparative method, emerged three categories: 1) Childhood: Stage of normality, without recognition of latent kidney disease, 2) Adolescence: an awakening stage when a health issue is confirmed, uncertainty about the life project and final acceptance of dialysis therapy as a way of survival and 3) Youth: transplantation, a hope for a life change.

Conclusion: The transition from childhood to youth in people with CKD, is a journey that begins in childhood, in disregard of the health problem; continues into adoles- 
cence with uncertainty for the construction of a life project given the dependence on dialysis therapy; and finally, in youth, with entry into kidney transplant program, it is transformed into a hope for a life change.

Key words: Transition, Uncertainty, Hope, Youth, Adolescents.

\section{RESUMEN}

Introducción: La transición a la juventud de niños y adolescentes con enfermedad renal crónica (ERC) trae consigo múltiples cambios que los profesionales de Enfermería deben incorporar a protocolos de cuidado para la promoción de la salud y prevención de la enfermedad renal.

Objetivo: Profundizar en el significado que tiene para personas en terapia dialítica haber vivido la transición infancia, adolescencia, juventud con ERC.

Metodología: Estudio cualitativo, siguiendo la Teoría Fundamentada en los datos. Participaron nueve personas con edades entre 18 y 24 años. La recolección de la información se realizó mediante entrevistas a profundidad y bajo criterios de credibilidad, auditabilidad y transferibilidad.

Hallazgos: A través de la codificación de datos, el muestreo teórico y el método comparativo constante, surgieron tres categorías: 1) Infancia: Etapa de normalidad, sin reconocimiento de una enfermedad renal latente, 2) Adolescencia: Etapa de despertar al comprobar un padecimiento, incertidumbre por el proyecto de vida y aceptación final de la terapia de diálisis como un camino de sobrevivencia y 3) Juventud con ERC: El trasplante, una esperanza para un cambio de vida

Conclusión: La transición de la infancia a la juventud en personas con ERC, es un camino que inicia en la niñez, con desconocimiento del problema de salud; continúa en la adolescencia con incertidumbre para la construcción de un proyecto de vida dada la dependencia de la terapia dialítica y finalmente, en la juventud, con el ingreso al programa de trasplante renal, se transforma en una esperanza para un cambio de vida.

Palabras clave: Transición, incertidumbre, esperanza, juventud, adolescentes.

\section{RESUMO}

Introdução: A transição da juventude de crianças para adolescentes com doença renal crônica (DCR), traz múltiplas mudanças no qual os profissionais de enfermagem devem incorporar aos protocolos de cuidado para promoção e prevenção da doença renal.

Objetivo: Compreender qual o sentido das pessoas em terapia de diálise que viveram a transição entre infância, adolescência e juventude com DRC.

Metodologia: Pesquisa qualitativa, seguindo a teoria fundamentada nos dados. Participaram nove pessoas com idades entre 18 e 24 anos. A coleta de informações foi realizada por meio de entrevistas detalhadas e sob critérios de credibilidade, auditabilidade e transferibilidade.

Resultados: Usando a codificação de dados, amostragem teórica e método comparativo constante, emergiram três categorias: 1) infância, etapa de normalidade, sem o reconhecimento de uma doença renal latente. 2) Adolescência: etapa de acordar ao verificar um sofrimento, incerteza pelo projeto de vida e aceitação final da terapia de diálise como um caminho de sobrevivência e 3) juventude com DRC: transplantação, uma esperança de mudança da vida. Conclusão: A transição da infância para juventude em pessoas com DRC 
é um caminho que começa na infância, com falta de conhecimento do problema de saúde; segue com adolescência, com incerteza para construção de um projeto de vida pela dependência da terapia de diálise e finalmente na juventude, com o ingresso ao programa de transplantação renal, torna-se uma esperança de mudança da vida.

Palavras-chave: Transição, incerteza, esperança, juventude, adolescentes.

\section{INTRODUCCIÓN}

La transición infancia, adolescencia $y$ juventud, conlleva una serie de cambios a nivel físico, psicológico, individual y social que requieren acompañamiento familiar $y$ una atención integral en salud para lograr resultados positivos en esta evolución. Si sumado a lo anterior, el niño o el adolescente, afronta una ERC, la demanda de los servicios de salud durante esta transición será mayor, implicando un esfuerzo extra de la familia y mayor apoyo del sistema de salud para mejorar el acceso y la efectividad de los servicios. Acorde al contexto educativo y social de cada persona, los profesionales de Enfermería deben lograr que estas transiciones sean saludables y las personas tengan una mejor calidad de vida. En este sentido, Afaf Meléis (citado en Alligood, MarrinerTomey, 2010), en su teoría sobre las Transiciones, conceptúa que los tipos de transiciones incluyen desarrollo, salud y enfermedad y que "las transiciones tienen condiciones que influyen en cómo una persona se mueve hacia ella, que facilitan o impiden el progreso para lograr una transición saludable y las mismas afectan las vidas de las personas y los trabajadores de salud" (citado en Alligood, Marriner-Tomey, 2010)
En el cuidado de una persona que vive una transición, los enfermeros deben conocer a la persona de una manera amplia para lograr una intervención terapéutica, enfocada desde la educación para la promoción y prevención, que permita crear condiciones óptimas para que esta transición sea saludable. Los resultados de dicha intervención, se pueden evidenciar entre otros, porque la persona logra un conocimiento amplio de su situación y encuentra la oportunidad de mejorar su calidad de vida a pesar de las dificultades que vive.

Con relación al problema de la ERC, estadísticas sobre la situación a nivel mundial, informan cerca de 1,6 millones de personas en hemodiálisis y 200.000 en diálisis peritoneal (Jain, Blake, Cordy y Gard, 2012, p. 533544). En países desarrollados, la incidencia de esta enfermedad, se encuentra entre 300 a 340 ppmh y se incrementa cada año en un 10\% (Alfonso de León, Gámez, Hay de la Puerta Zoto, et al. 2013). La situación en Latinoamérica es bastante preocupante debido al incremento de la incidencia y prevalencia de enfermedades crónicas como la diabetes y la hipertensión arterial y a que un alto porcentaje de personas desconoce su diagnóstico, aunado a la falta de detección oportuna y seguimiento adecuado que evite o retrase complicaciones como la Insuficiencia Renal Crónica Terminal (IRCT). En Latinoamérica, la prevalencia de la ERC es de 447 ppmh, y la incidencia es de 147 ppmh (Alfonso de León et al., 2013). La distribución de la prevalencia por países en la región es así: Puerto Rico: 1026, Cuba: 225, Argentina: 667, Brasil: 485 y Bolivia 63 (Almaguer, Magrans y Herrera. 2009).

En Colombia, para el 2014, la Cuenta de Alto Costo (CAC, 2014), informó 770.428 personas con diagnóstico de ERC. Para este mismo año se presentaron 29.314 casos de ERC, 


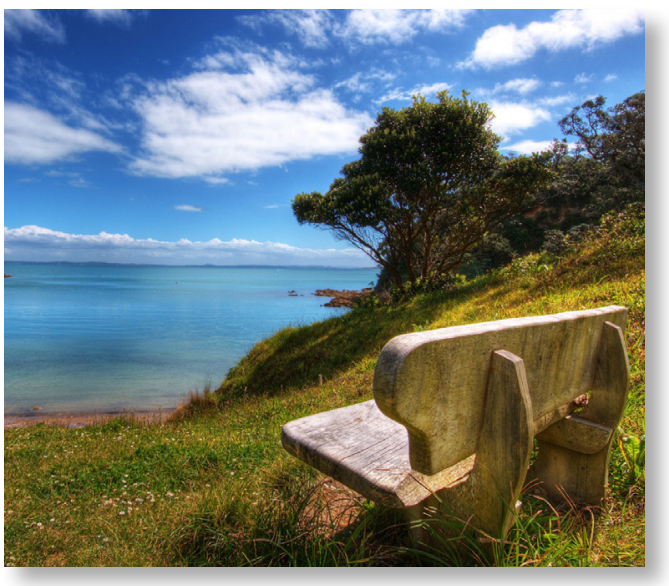

cifra que se ha aumentado considerablemente según el informe de seguimiento desde el año 2008. Del total de personas que padecen ERC, el 56,5\% se encuentran en hemodiálisis (HD), $23,2 \%$ en diálisis peritoneal (DP) y $17,8 \%$ han recibido trasplante renal. El número de casos en personas jóvenes, hasta los 24 años de edad, muestra también un importante incremento; se registran alrededor de 700 casos en terapia de reemplazo (TRR), de los cuales, solo 188 personas han recibido trasplante renal (CAC, 2014).

Por otro lado, para agravar esta problemática, la presentación de la sintomatología renal se da cada vez más en edades tempranas de la vida, debido a factores hereditarios, interacción de factores que tienen relación con la economía, educación, acceso a servicios de salud y saneamiento entre otros (Alfonso de León et al., 2013); sin embargo, su diagnóstico sigue siendo tardío y como consecuencia, los resultados para la salud son negativos y la transición de la infancia a la juventud se ve vulnerada porque esta población, además de los procesos normales que se producen en estas etapas, debe afrontar y adaptarse a procesos de salud-enfermedad.

Dentro de estas adaptaciones, el niño y el adolescente se ve expuesto a cambios rela- cionados con su imagen corporal, identidad sexual, relaciones sociales, apego emocional, educación/vocación, ajustes a las demandas de la sociedad, que espera madurez en el comportamiento y la internalización de una serie de valores con el fin de asumir el papel de adulto (O’Sullivan-Oliveira, Fernandes, Borges, Fishman, 2014).

Una revisión sistemática reciente acerca de las "Experiencias de los niños en diálisis" (Tjaden, Tong, Henning, Groothoff, y Craig, 2012), muestra cómo los niños con ERC, diagnosticada a temprana edad, con frecuencia sufren alteraciones y retraso en su crecimiento y desarrollo, estatura baja, enfermedades como la enfermedad mineral ósea, hospitalizaciones frecuentes, episodios de infección del acceso vascular o peritoneal para diálisis, restricción en la dieta y líquidos orales, uso de múltiples medicamentos en su manejo, cambios en estilo de vida e incluso de domicilio para acceder a la terapia, entre otros aspectos que pueden ocasionar pérdidas que los niños y adolescentes deben afrontar: separación del grupo de amigos, abandono escolar por terapias u hospitalizaciones, dependencia de sus padres o cuidadores, incertidumbre sobre el trasplante, sobre la vida laboral, sobre su futuro.

El profesional de enfermería en el nivel I de atención, que lidera los programas de Crecimiento y Desarrollo y de Escolares y Adolescentes, tiene la responsabilidad de llevar a cabo los programas de promoción y prevención, que permitan la detección oportuna de alteraciones renales y una remisión temprana de los niños a fin de intervenir y evitar complicaciones y promover que la transición infancia - juventud se haga de una manera saludable.

Teniendo en cuenta que a uno de los programas de trasplante renal de la ciudad de Cali Colombia, asisten jóvenes que han vivi- 
do desde niños con enfermedad renal, que se convirtió en crónica y todos ellos están en terapia dialítica y adscritos al programa de trasplante, donde la transición infancia-juventud se ha visto alterada, es importante tener como fuente de conocimiento el significado que para estas personas ha tenido el transcurrir su infancia, adolescencia y juventud con tratamientos farmacológicos diversos, asistencia a instituciones de salud de manera frecuente, hospitalizaciones, terapias de reemplazo renal y finalmente la adscripción a un programa de trasplante como único camino para lograr trasplantarse y mejorar su salud, se pretendió con esa investigación, identificar y profundizar en los aspectos no develados de esta transición, relacionándolos con los postulados que plantean dos teóricas de enfermería, como son Afaf Meléis, en su Teoría de las Transiciones y Merle Mishel en la Teoría de la Incertidumbre. Con los hallazgos se busca contribuir a que los protocolos de enfermería para el cuidado de las personas con ERC estén orientados a favorecer una transición infancia adolescencia y juventud saludable.

\section{MATERIALES Y MÉTODOS}

Tipo de estudio: Estudio cualitativo, para el cual se utilizó la Teoría Fundamentada en los datos (Guedes, Alacoque, Macedo de,Marcelino de Melo, Schaefer, y Josete Luzia, 2016), la cual permite comprender e interpretar los significados que ha tenido para un grupo de jóvenes, la experiencia de vivir la infancia, adolescencia y juventud con ERC. El rigor metodológico del estudio se obtuvo bajo los criterios de credibilidad, confirmabilidad y transferibilidad.

Muestra: Se obtuvo por saturación de datos con nueve participantes que cumplieron con los siguientes criterios de inclusión: edad entre 18 y 24 años, diagnóstico de ERC y encontrarse en protocolo de evaluación pretrasplante o en lista de espera en el Programa de Trasplante Renal de una Institución de salud de cuarto nivel de la ciudad de Cali, Colombia.

Aspectos Éticos: El estudio fue aprobado por el Comité de Ética de la Universidad del Valle, mediante Acta No. 010-014 del 10 de julio de 2014 y por el Comité de Ética de la Institución de Salud de cuarto nivel en donde se desarrolló la investigación, con clasificación de bajo riesgo.

\section{Recolección y análisis de la información:} Se requirió 12 meses para la recolección y análisis de la información, la cual se realizó en las siguientes etapas:

1. Revisión de historias clínicas de las personas adscritas al programa y que cumplieran con los criterios de inclusión, con el fin de hacer la caracterización sociodemográfica y clínica.

2. Reunión inicial con los jóvenes que aceptaron participar en el estudio, en donde se informó los objetivos, metodología y utilidad de los resultados. Se obtuvo la firma del consentimiento informado, en el cual se incluyó el consentimiento de grabación.

3. La recolección y análisis de la información se realizaron simultáneamente. Se efectuaron en promedio dos entrevistas en profundidad a cada participante, las cuales fueron grabadas y transcritas textualmente por uno de los investigadores. Las mismas tuvieron una duración entre 45 y 90 minutos y se acordó con ellos la fecha, hora y sitio para la realización de las mismas. Se protegió la identidad y los relatos de los participantes, mediante el uso de nombres ficticios.

Para el desarrollo de las entrevistas, se elaboró una guía con preguntas orientadas a indagar sobre el significado que tenía para la 
persona su estado de salud, autopercepción de su vida desde la niñez hasta la juventud padeciendo ERC; sentimientos al momento de conocer el diagnóstico, el tratamiento de la enfermedad, la terapia dialítica y la espera del trasplante renal; y finalmente, el significado y la influencia que estos cambios tuvieron en su vida a nivel físico, familiar, psicológico y social desde la niñez, adolescencia y en su momento actual de juventud. Las preguntas que inicialmente fueron abiertas se volvieron más específicas a medida que la investigación fue avanzando.

El análisis de los datos se efectuó, siguiendo los pasos metodológicos de la Teoría Fundamentada. Inicialmente se efectuó la descripción y análisis línea a línea de las entrevistas y notas de campo, estableciendo códigos en vivo y sustantivos, los cuales se analizaron a través de preguntas teóricas y comparaciones. Los datos se organizaron, interpretaron e integraron en conceptos, subcategorías y categorías de acuerdo a las propiedades, dimensiones y relaciones entre ellos, encontradas en los datos. También se utilizó la codificación axial, utlizando el Modelo Paradigma para estructurar los datos de una manera sistemática, analizando las circunstancias en las cuales surgieron los acontecimientos que les fueron más significativos a los participantes en su transición niñez adolescencia y juventud con un diagnóstico de ERC, así como la respuesta, a través del tiempo, a su problema, de las personas, instituciones de salud y comunidades, $y$ las consecuencias o resultado que obtuvieron de esas acciones o interacciones.

Relacionar las subcategorías con las categorías que emergieron de los datos, permitió a los investigadores tener una explicación más profunda y develar el significado que tuvo para los participantes, vivir desde la infancia hasta la juventud, etapa que se encontraban viviendo en ese momento, con diagnóstico de ERC.

\section{RESULTADOS}

Los hallazgos de la investigación se presentan en dos partes. En la primera de ellas, se describe el perfil sociodemográfico de los participantes, antecedentes de la enfermedad, así como algunos datos significativos de los padres o cuidadores. En la segunda parte, se presentan las subcategorías y categorías que surgieron de los datos. Para la presentación de los datos se utilizaron nombres ficticios.

\section{Aspectos socioeconómicos}

La edad promedio en la que los participantes iniciaron diálisis fue de 18 años. Ocho de los nueve participantes tuvieron afiliación a un sistema de salud subsidiado en el momento del diagnóstico. Solo una participante contaba con afiliación a una Entidad Promotora de Salud (EPS), como beneficiaria de su padre adoptivo. (Ver Tabla 1).

Cuatro de los participantes son procedentes del área rural, no tenían acceso a consulta médica y refirieron que cuando la tuvieron en su niñez, las evaluaciones del grupo médico nunca indicaron controles o seguimientos. En uno de los casos, el acceso posible fue donde un "yerbatero".

Todos los participantes son personas de escasos recursos, dependen económicamente de sus padres, cuyo ingreso familiar no supera dos salarios mínimos. Los jóvenes manifiestan gran deseo de estudiar y trabajar, sin embargo, el tiempo invertido en su terapia y en su salud y la escasez de recursos económicos no se los permitió. La economía familiar debió redistribuirse para cubrir gastos de transporte para asistir a la terapia, para exámenes, me- 
dicamentos, interconsultas, que en algunos casos no lograron conseguir oportunamente a través de su entidad de salud.

Tabla 1. Perfil sociodemográfico de los participantes

Al momento del diagnóstico de ERC, los participantes se encontraban cursando estudios de secundaria o se habían graduado recientemente; 7 de ellos obtuvieron su título de bachiller y en ninguno de los casos tuvieron la posibilidad de continuar estudios técnicos o profesionales.

\section{Categorías}

Se obtuvieron tres categorías con sus respectivas subcategorías (Figura 1), las cuales surgen de las propiedades y relaciones encontradas en cada una de ellas y que muestran cual fue el significado de transitar por cada una de las etapas Infancia, Adolescencia y Juventud para los jóvenes participantes del estudio. Estas son:

2.1 Infancia: Etapa de normalidad, sin reconocimiento de una enfermedad renal latente.

2.2. Adolescencia: Despertar para el reconocimiento de un padecimiento, incertidumbre por el proyecto de vida y aceptación final de la terapia de diálisis como un camino de sobrevivencia

2.3 Juventud con ERC: El trasplante, una esperanza para un cambio de vida.

$\mathrm{Al}$ interrelacionar estas tres categorías, surge la categoría central, la cual puede considerarse una teoría sustantiva fundamentada en los datos:

"La transición de la infancia a la juventud en personas con enfermedad renal crónica, es un camino de incertidumbre, pero de esperanza para un cambio de vida con el trasplante renal".

A continuación se describen cada una de las categorías:

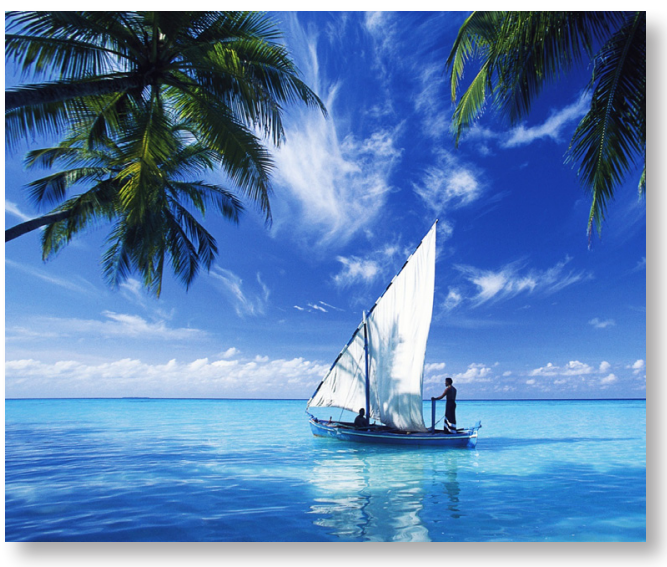

2.1 Infancia: Etapa de "normalidad", sin reconocimiento de una enfermedad renal latente.

De acuerdo con el análisis de los datos, ésta etapa transcurrió con "normalidad"; los participantes la vivieron como niños "normales", asistieron a la escuela y realizaron actividades que cotidianamente hacen los infantes. Sin embargo al profundizar en los datos, los participantes recuerdan que desde su niñez presentaron signos y/o síntomas, probables premonitorios de enfermedad, constituidos principalmente por "dolor de cabeza", "ronchas en la piel", "salida de sangre por la nariz", "hinchazón de los pies", "fiebre alta", "anemia”, "vómito", "mareo", "cansancio", "mal genio", los cuales fueron percibidos como frecuentes por todas las personas entrevistadas. Uno de los participantes lo refiere de la siguiente manera:

"De mi niñez recuerdo que jugaba bastante, que bailaba, era feliz! y pues desde que me empezó a salir unas ronchas yo me sentía mal; mi mamá me llevaba $\mathrm{pa}^{\prime}$ un lado y $\mathrm{pa}^{\prime}$ otro, tenía como 8 año y nunca me hicieron nada, decían que era alérgica, tenía la presión muy alta, me mandaban algo y pues ya me pasaba. Cuando tenía los 16 años me puse mal, tenía la hemoglobina en 5, me tuvieron hospitalizada como 2 meses, me dijeron que estaba sufriendo de los riñones y que me iban a hacer diálisis, yo ni sabía qué era eso" 
En todos los participantes nunca se realizó un seguimiento médico ni por otros profesionales de la salud, tampoco se ordenaron laboratorios ni remisiones a especialistas y no fueron detectados ni seguidos en programas de Enfermería como los de Crecimiento y Desarrollo, Escolar y Adolescente. Tras algunos años, la situación de salud se agudizó, debiendo consultar a un servicio de urgencias, en donde determinaron el diagnóstico y la necesidad inmediata de iniciar el tratamiento con terapia de reemplazo renal.

\subsection{Adolescencia: "Despertar al comprobar} un padecimiento, incertidumbre por el proyecto de vida y aceptación final de la terapia de diálisis como camino de sobrevivencia”.

Se encontró que los nueve participantes del estudio presentaron sintomatología de una enfermedad crónica y trascendieron a la adolescencia debiendo enfrentar el momento en que la enfermedad renal se expresa como urgencia, con necesidad de iniciar una terapia de diálisis. Asumir una terapia crónica, fue para ellos, trascender de un estado de "salud" a uno de "enfermedad". Una vez establecido el diagnóstico, el afrontamiento inicial fue descrito por los jóvenes, como un estado de "shock", con profundos sentimientos de depresión, frustración, soledad, negación de la enfermedad, desesperanza, rebeldía. Manifestaron la aparición de sentimientos de pérdida de la salud, incertidumbre por el diagnóstico, dependencia, esclavitud; percibieron cambios físicos, en la autoimagen y autoestima, desinterés, falta de adherencia al tratamiento. Ahora debían asistir periódicamente a una terapia para poder vivir y esto implicaba innumerables cambios y restricciones en la cotidianidad de sus vidas como adolescentes. Ellos desconocían sobre el diagnóstico, algunos buscaron información a través de internet o simplemente fueron aprendiendo a medida que transcurría su vida en diálisis. Al respecto uno de los jóvenes manifiesta:

"Me dijeron que me tenían que hacer un tratamiento, yo jamás me imaginé que era eso, pues...yo dije: ¡ah sí!, pastas, pero no, cuando ya me subieron a la unidad me dijeron: mira, te vamos a poner un catéter...yo prácticamente entré en shock, yo no los escuché, yo me puse a llorar".

Los jóvenes manifestaron haber sido rechazados por parte de empleadores al momento de aspirar a una vacante. Ocho de los participantes manifestaron que tuvieron algunos empleos temporales y ninguno de ellos tuvo un trabajo estable con las prestaciones sociales obligatorias.

Los jóvenes refirieron, que al reconocer su padecimiento, comprendieron lo que implicaba en sus vidas: ahora su vida giraba alrededor de la diálisis y su proyecto de vida era realmente una incertidumbre al igual que para sus familiares quienes reorganizaron prioridades, tareas y funciones para poder ser el soporte que ellos necesitaban en ese momento. Al respecto uno de los jóvenes manifiesta:

"Mientras estuve en el hospital pensaba que cuando volviera a casa iba a ir al colegio; cuando empecé a dializarme en peritoneal, entendí que mi vida había cambiado cien por ciento, entendí que tenía que depender de una diálisis, que estaba amarrado a algo, entonces ahí sí me deprimí, ahí si fue fuerte".

$\mathrm{Al}$ inicio, cuando los jóvenes conocieron el diagnóstico y la comprensión de la enfermedad era superficial, aceptar la terapia y ser adherente a ella, fue un proceso largo y complejo, ya que también debieron aceptar que sin ella no podían vivir. Al respecto el participante comenta: 
"Yo lloraba haciéndome diálisis, yo no me tomaba las pastillas, todo era molesto para mí; entonces me molestó que tanto me molestara, me fastidió mantener así molesta, entonces más bien comencé a aceptar la diálisis, a tomarme mis medicamentos y me di cuenta de que las cosas mejoraban, entonces comencé a mejorar, y ya es mejor mi actitud.".

Los jóvenes entrevistados también manifestaron que el soporte de los grupos de salud puede ser mayor y mejor y ayudarles de diferentes maneras, debido a que las necesidades de ellos son diversas e incluye la detección oportuna, el seguimiento médico, orientación educativa, laboral, apoyo económico, espiritual, entre otros.

\subsection{Juventud con ERC: "El trasplante, una es-} peranza para un cambio de vida”.

De acuerdo con los datos analizados, cuando los adolescentes trascendieron a la etapa de la juventud y fueron remitidos al "protocolo de evaluación pretrasplante renal", la posibilidad de trasplantarse y dejar de depender de la diálisis, se convirtió para ellos, en una "esperanza para un cambio de vida”. El trasplante fue concebido por los jóvenes como la mejor terapia; consideraban que una vez trasplantados, podrían estudiar, trabajar, hacer muchas cosas que dejaron de hacer por el estado de salud y la terapia de diálisis. Al respecto uno de los participantes manifiesta:

"Porque uno trasplantado ya no tiene que estar en la terapia, ya tiene más tiempo, ya se siente uno mejor, tanto física como emocionalmente, uno ya con el trasplante está más tranquilo, fuera de eso, no tanto uno sino la familia que ya lo ve mejor".

Igualmente, los jóvenes manifestaron incertidumbre por el tiempo de espera para ser llamados al trasplante. Algunos pensaron que esta intervención se daría pronto, una vez terminaran la evaluación clínica; sin embargo, el ingreso a la lista, terminó por aclararles que se realizaría una vez que apareciera un donante compatible y esto podría ser cuestión de días, meses o años.

La categoría central devela que "La transición de la infancia a la juventud en estas personas jóvenes con un camino recorrido de tantos años con el padecimiento de ERC, está cargado de incertidumbre, pero de esperanza para un cambio de vida con el trasplante renal". Estos hallazgos demandan de los profesionales de Enfermería, un cuidado integral que involucre a la familia, la sociedad y los equipos de salud que los atienden, con el fin de que la transición en el ciclo evolutivo y del estado de salud al de enfermedad, se haga de manera saludable y permita una buena calidad de vida en este grupo de personas.

Figura 1. Significado de la Transición Infancia adolescencia juventud en personas con ERC.

\section{DISCUSIÓN}

Para los jóvenes participantes en el estudio, la "infancia” significó “felicidad", una etapa en que jugaban e iban a la escuela como "todos los niños", una etapa que se percibió como "normal", hasta cuando aparecen los signos y síntomas probables premonitorios de la enfermedad, pero sobre los cuales no se hizo un seguimiento que permitiera la detección temprana y la prevención o retraso de la IRCT.

Son diversos los factores que intervienen para que suceda este tipo de situaciones en nuestro medio, entre ellos se encuentra la dificultad de acceso a la seguridad social en salud, bajo nivel educativo de sus padres, bajos recursos económicos, falta de una política real, enfocada a la educación a la promoción de la salud y prevención de la enfermedad. Este ha- 
llazgo es similar a lo encontrado en Colombia por Martínez, Ordoñez y García (2007), que si bien no fue realizado con pacientes adolescentes, evidenció las graves deficiencias en la atención de personas diabéticas, tanto en la detección temprana como en el tratamiento oportuno. Se trata de un estudio retrospectivo - descriptivo, en el cual analizaron los casos clínicos de 241 pacientes, que ingresaron a terapia de diálisis entre 2003 y 2005. En este grupo de pacientes, tras el diagnóstico de diabetes, transcurrieron solo 13 años para desarrollar enfermedad renal y solo transcurrió medio año para el ingreso a terapia de reemplazo renal, es decir que "el diagnóstico es tardío y los pacientes acceden al mismo, cuando el daño renal es irreversible y rápidamente progresivo" (Martínez et al., 2007). Hallazgos similares reportan los estudios de: Jungers et al., (2006) y el Estudio de Cohorte Nacional Danés (Hommel, Madsen y Kamper 2012). El primer estudio trata la problemática de la referencia tardía de los pacientes con ERC, causas, consecuencias, y enfoques para mejorar, y en el segundo trabajo, se evidencia la importancia de la derivación temprana para el tratamiento de la ERC. Al respecto, el presente estudio encontró que aunado a que la detección de la enfermedad renal fue tardía, el diagnóstico se realizó en una situación de emergencia, cuando se requirió de inmediato el inicio de terapia de diálisis. Es decir, que en el caso de los nueve participantes, no hubo detección temprana ni seguimiento oportuno, la enfermedad renal no se diagnosticó ni se retrasó, hizo su curso natural. Adicional a esto, el hecho de llegar en un estado avanzado de su enfermedad, dificulta establecer la causa de la patología, ya que métodos diagnósticos como la biopsia renal no podrán ofrecer información certera y es mayor el riesgo de realizar dicho procedimiento ante la uremia elevada y la atrofia del tejido renal.

Por otra parte, los signos y síntomas que aparecieron durante la infancia, se muestran en la adolescencia en el contexto de una enfermedad que se estableció como crónica y que requirió una terapia de diálisis. Para los jóvenes del estudio, conocer el diagnóstico y despertar al reconocimiento de su padecimiento, significó un cambio inesperado en su vida, que trajo consigo incertidumbre por el futuro, cambios físicos y fisiológicos, dependencia de la terapia, dificultad para estudiar y trabajar. En este sentido, el estudio concuerda con los hallazgos del trabajo realizado en Colombia por Morales y Castillo (2007), en el que se utilizó la fenomenología interpretativa para describir las Vivencias de los(as) adolescentes durante el proceso de diálisis, encontrando que "los adolescentes con Insuficiencia Renal Crónica, se sienten preocupados y con frecuencia insatisfechos con su apariencia física, por los cambios fisiológicos y morfológicos que les ocurren; luchan por su independencia y mantienen una búsqueda constante de su identidad personal; enfrentan el desafío de una enfermedad crónica, que representa retos y estrés adicionales" (Morales y Castillo, 2007).

Por otra parte, a medida que estos niños y adolescentes, trascienden a la etapa de la juventud, su pensamiento fue madurando $\mathrm{y}$ con ello adquirieron mayor responsabilidad y disciplina frente a su terapia, aceptando mejor los cambios para un adecuado control de su enfermedad; organizaron su cotidianidad, con la esperanza de que tras el trasplante, su proyecto y calidad de vida serían mejores al dejar de depender de la máquina de diálisis y tener menores restricciones en aspectos nutricionales, de ingesta de líquidos y sentirse en con- 
diciones de trabajar, estudiar y participar en actividades recreativas y de bienestar. En este sentido, el estudio sobre "Características del estado emocional de los pacientes con enfermedad renal crónica" (Gonzáles, Herrera, Romero y Nieves, 2011), encontró también, que los pacientes más jóvenes, "manifiestan cierta resistencia a aceptar su situación. Sin embargo, en sus expresiones predomina el deseo de vivir, de "seguir luchando" y la esperanza del éxito en el trasplante" (Gonzales, et al., 2011).

Merle Mishel (2013), teórica de enfermería, concibe que la incertidumbre está presente a lo largo del camino que recorren los niños y adolescentes a partir del diagnóstico de ERC. Incertidumbre por un futuro desconocido, por un proyecto de vida que en algún momento se planeó o se imaginó y el choque con su momento real a partir del diagnóstico.

El cuidado de enfermería y en general, de los equipos de salud multidisciplinarios, deben lograr que la incertidumbre, como lo conceptúa Mishel, se convierta en la oportunidad para lograr mejorar el estado de ánimo de la persona enferma, mediante el apoyo social y del personal de salud que le atiende. La transición en el ciclo evolutivo y en el proceso de salud/enfermedad de este grupo de personas, requiere el acompañamiento de la familia, la sociedad y los equipos de salud. Afaf Ibrahim Meléis, teorista en enfermería, conceptúa que los enfermeros pueden contribuir a que las transiciones sean saludables. Esto es posible lograrlo preparando a las personas, previo a la transición (Melesis, 2010).

En concordancia con los hallazgos de este estudio, el enfermero Cura (2012), realizó un estudio fenomenológico acerca de la transición de la adolescencia a la adultez de personas con enfermedad renal terminal, con edades entre los 17 y 22 años, y recomienda, que en la práctica de enfermería es de gran importancia "la escucha al paciente y la educación sanitaria con el propósito de que ellos tengan un papel activo en su autocuidado y en la autogestión de su proceso" (Cura, 2012., p. 118-123).

En los niños y jóvenes, aspectos como la adherencia a las terapias de reemplazo renal, a los protocolos de seguimiento, medicación y cuidados en pre y post trasplante, pueden verse influenciados por cambios que ocurren durante la transición a la edad adulta y afectar la salud y la vida.

La revisión sistemática realizada por Haavisto et al., (2013., p. 256-265), sobre los “factores de riesgo para el deterioro de la calidad de vida y ajuste psicosocial después del trasplante pediátrico de corazón, riñón, concluye que el proceso de transición de los pacientes pediátricos se ha asociado a altas tasas de no adherencia al tratamiento, situación que también se evidenció en los participantes del presente estudio. El estudio realizado por Annunziato $\mathrm{R}$, et al. (2010., p. 823-829) concluye que es necesario preparar a los jóvenes y padres desde antes de la transferencia del servicio pediátrico al de adultos, en todo lo relacionado con el conocimiento sobre la medicación, autocuidado, citas médicas de control y diligencias administrativas, entre otros aspectos, ya que hasta el momento, la mayor parte de este proceso lo realizan principalmente los padres o cuidadores, por lo que esta preparación será clave para contribuir a aumentar la responsabilidad y adherencia de los adultos jóvenes.

\section{CONCLUSIONES}

La transición de la infancia a la juventud en personas con ERC, es un camino que inicia tempranamente en la niñez, con desconocimiento de este problema de salud; continúa en la adolescencia con incertidumbre y duda 
para la construcción de un proyecto de vida que se torna sombrío ante la terapia dialítica, pero que en la juventud, se transforma en una esperanza para un cambio de vida, con el ingreso al programa de trasplante renal

Profundizar en el significado que tiene para los sujetos de cuidado, en este caso jóvenes con ERC, el periodo de transición de la infancia a la juventud, comprendida entre el diagnóstico de la enfermedad y la espera de trasplante renal, permite al equipo de salud, comprender los diferentes aspectos sobre los cuales se deben fundamentar los protocolos de cuidado para la promoción de la salud, prevención de la enfermedad renal y adherencia a las terapias, con el fin de contribuir a que estas personas, desde la niñez, tengan una mejor calidad de vida a través de la detección y seguimiento oportuno que permitan disminuir la elevada incidencia de la enfermedad y retrasar las complicaciones tanto en el pre como en el postrasplante renal.

\section{BIBLIOGRAFÍA}

- Alligood, M. R., \& Marriner-Tomey, A. (2010). Nursing Theorists and Their Work. Maryland Heights, Mo: Mosby.

- Alfonso de León, J.A., Gámez Jiménez, A., Hay de la Puerta Zoto, M., et al. (2013). Enfermedad renal crónica en el adulto mayor. Rev. Med. Electrón., 35(4), 306-318. Retrieved from http://scielo.sld.cu/scielo.php?script=sci $\underline{\operatorname{arttext} \& p i d=S 1684-18242013000400001 \& l a n g=p t}$

- Almaguer LM, Magrans BCh, Herrera V. (2009). Definición y estratificación de la enfermedad renal crónica, medición de la función renal, epidemiología, clínica, prevención y tratamiento. La Habana: Instituto de Nefrología. 2009.

- Annunziato, R. A., Hogan, B., Barton, C., Miloh, T., Arnon, R., Iyer, K., \& Kerkar, N. (2010). A translational and systemic approach to transferring liver transplant recipients from pediatric to adult-oriented care settings. Pediatric Transplantation, 14(7), 823-829. doi:10.1111/

\section{j.1399-3046.2010.01348.x}

- Cuenta de Alto Costo. Situación de la Enfermedad Renal Crónica en Colombia. Fondo Colombiano de Enfermedades de Alto Costo. Bogotá, D.C. Colombia.

- Cura, J. (2012). Interpreting transition from adolescence to adulthood in patients on dialysis who have end-stage renal disease. Journal of Renal Care, 38, 3, 118-123.

- Jain, A. K., Blake, P., Cordy, P., \&amp; Garg, A. X. (2012). Global trends in rates of peritoneal dialysis. Journal of The American Society Of Nephrology: JASN, 23(3), 533544. doi:10.1681/ASN.2011060607

- Guedes dos, S., Alacoque Lorenzini, E., Francisca Georgina Macedo de, S., Gabriela Marcelino de Melo, L., Ana Lúcia Schaefer Ferreira de, M., \& Josete Luzia, L. (2016). Perspectivas metodológicas para o uso da teoria fundamentada nos dados na pesquisa em enfermagem e saúde / Metho. doi:10.5935/1414-8145.20160056

- Gonzáles, Y., Herrera, L. F., Romero, J. L. \& Nieves, Z. (2011) Características del estado emocional en pacientes con enfermedad renal crónica. Revista PsicologiaCientifica.com, 13(20). 1 de diciembre de 2011. Disponible en: http://www.psicologiacientifica.com/enfermedad-renalcronica-pacientes-estado-emocional.

- Haavisto, A., Korkman, M., Sintonen, H., Holmberg, C., Jalanko, H., Lipsanen, J., \& Qvist, E. (2013). Risk factors for impaired quality of life and psychosocial adjustment after pediatric heart, kidney, and liver transplantation. Pediatric Transplantation, 17(3), 256-265. https://doi. org/10.1111/petr.12054

- Hommel, K., Madsen, M., \& Kamper, A. (2012). The importance of early referral for the treatment of chronic kidney disease: a Danish nationwide cohort study. BMC Nephrology, Vol 13, Iss 1, P 108 (2012), (1), 108. doi:10.1186/1471-2369-13-108

- Jungers, P., Joly, D., Nguyen-Khoa, T., Mothu, N., Bassilios, N., \& Grünfeld, J. (2006). Article original: Retard persistant au suivi néphrologique de l'insuffisance rénale chronique. Causes, conséquences et moyens d'amélioration. La Presse Medicale, 35(Part 1), 17-22. doi:10.1016/S0755-4982(06)74514-6

- La salud de los jóvenes: un desafío para la sociedad, (2000). Informe Salud para todos en el año 2000 . Serie 


\section{Cultura de las Cuidados}

de Informes Técnicos. Organización Mundial de la Salud. ISBN 9243207318

- Martínez, F. L., Ordoñez, I. E., \& García, D. L. (2007). Deficiencias en el tratamiento de pacientes diabéticos que terminaron en enfermedad renal crónica. Acta Medica Colombiana, (2), 57

- Meleis, A. I. (2010). Transitions theory: Middle-range and situation-specific theories in nursing research and practice. New York: Springer Pub. Co.

- Mishel, M. (2013). Theories of Uncertainty in Illness. Middle Range Theory For Nursing.

- Morales, L. C., \& Castillo, E. (2007). Vivencias de los (as) adolescentes en dialisis: Una vida de multiples perdidas pero con esperanza. Colombia Medica, 38(4 SUPPL. 2), 44-53.

- O'Sullivan-Oliveira, J., Fernandes, S. M., Borges, L. F., Fishman, L. N., Sullivan-oliveira, J. O., \& Fernandes, S. M. (2014). Transition of Pediatric Patients to Adult Care : An Analysis of Provider Perceptions Across Discipline and Role. Pediatric Nursing, 40(3), 113-120,142.

- Tjaden, L., Tong, A., Henning, P., Groothoff, J., \& Craig, J. C. (2012). Children's experiences of dialysis: a systematic review of qualitative studies. Archives Of Disease In Childhood, (5), 395

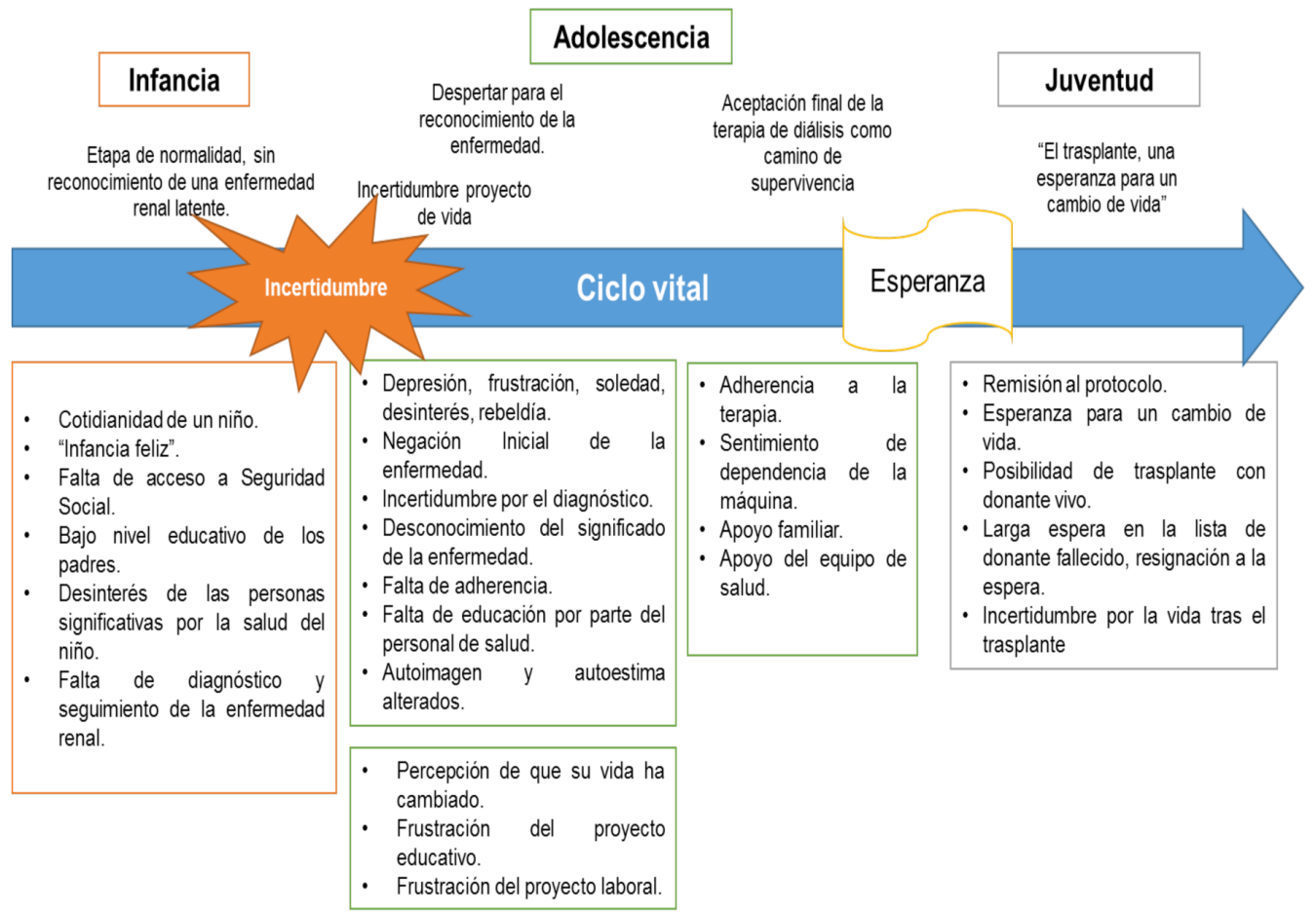

Figura 1. Categorías. Significado de la Transición Infancia adolescencia juventud en personas con ERC 


\section{ᄃultura de las Cuidados}

\begin{tabular}{|c|c|c|c|c|c|}
\hline Participante & $\begin{array}{c}\text { ERC } \\
\text { estadio clínico y } \\
\text { causa }\end{array}$ & $\begin{array}{c}\text { Edad de inicio signos } \\
\text { y/o síntomas }\end{array}$ & $\begin{array}{l}\text { Edad de Dx } \\
\text { e inicio de diálisis }\end{array}$ & Procedencia & $\begin{array}{c}\text { Familiar con quien convive, nivel } \\
\text { educativo y ocupación }\end{array}$ \\
\hline Samuel & $\begin{array}{l}\text { EC } 5 \text { de causa } \\
\text { desconocida }\end{array}$ & 8 años & 16 años & Rural & Madre: primaria/. Oficios varios. \\
\hline Silvia & EC 5 sec. a LES & 15 años & 21 años & Rural & $\begin{array}{l}\text { Madre primaria/ama de casa) } \\
\text { Padre: pensionado }\end{array}$ \\
\hline Linda & secundaria a LES & 16 años & 16 años & Urbana & $\begin{array}{l}\text { Padre: primaria/ conductor } \\
\text { Madre fallecida }\end{array}$ \\
\hline Beatriz & $\begin{array}{l}\text { EC } 5 \text { Síndrome } \\
\text { nefrótico }\end{array}$ & 18 años & 19 años & Urbana & $\begin{array}{l}\text { Madre adoptiva: Primaria/ama de } \\
\text { casa. Padre adoptivo Primaria/ } \\
\text { ebanista }\end{array}$ \\
\hline Mónica & causa desconocida & 10 años & 19 años & Urbana & Madre: Bachiller/ama de casa \\
\hline Sofía & causa desconocida & 3 años & 16 años & Urbana & Esposo: Bachiller/ Comerciante \\
\hline Lucia & causa desconocida & 18 años & 19 años & Urbana & Esposo: Bachiller/ Obrero \\
\hline Ángela & causa desconocida & 10 años & 15 años & Rural & Madre: Primaria/ oficios domésticos \\
\hline Natalia & secundaria a LES & 16 años & 20 años & Rural & Madre: Primaria/ oficios varios \\
\hline
\end{tabular}

Tabla 1. Perfil sociodemográfico de los participantes.

Fuente: Entrevistas a los participantes e Historias Clínicas Programa de Trasplante Renal Institución de salud nivel IV. Cali, Colombia.

ERC: Enfermedad Renal Crónica. EC: Estadío Clínico. LES: Lupus Eritematoso Sistémico. 